\title{
Maxillary Sinus Cancer pT4a TNM Finding v7
}

National Cancer Institute

\section{Source}

National Cancer Institute. Maxillary Sinus CancerpT4a TNM Finding v7. NCI Thesaurus. Code C89096.

Maxillary sinus cancer with moderately advanced local disease. T umor invades anterior orbital contents, skin of cheek, pterygoid plates, infratemporal fossa, cribriform plate, sphenoid or frontal sinuses. (from AJCC 7th Ed.) 